\title{
Bidirectional Screening of Diabetes Mellitus and Tuberculosis in Cambodia
}

\author{
Soy Ty Kheang ${ }^{1,2,3^{*}}$, Huot Theang ${ }^{1}$, Khun Kim Eam ${ }^{4}$, Mao Tan Eang ${ }^{4}$, Sok Kong ${ }^{5}$, Chhun Loun ${ }^{5}$, Aida \\ Olkkonen $^{6}$, Hala Jassim AlMossawi ${ }^{6}$, Neeraj Kak ${ }^{6}$
}

${ }^{1}$ Center for Health and Social Development (HSD), Phnom Penh, Cambodia; ${ }^{2}$ University Research Co., LLC (URC), Phnom Penh, Cambodia; ${ }^{3}$ School of Public Health, National Institute of Public Health (NIPH), Phnom Penh, Cambodia; ${ }^{4}$ National Centre for Tuberculosis and Leprosy Control (CENAT), Phnom Penh; ${ }^{5}$ Department of Preventive Medicines, Phnom Penh, Cambodia; ${ }^{6}$ Centre for Innovations and Technology, University Research Co., LLC (URC), Chery Chase, MD, United States

\begin{abstract}
Background: Cambodia has a high burden of Tuberculosis (TB) with an incidence rate of 326 per 100,000 population in 2018 and rapidly increasing rates of Diabetes Mellitus (DM) with prevalence rate 9.6\% in 2016. The introduction of the first national guidelines for the management of TB/DM co-morbidity in 2014 has resulted in the introduction of coordinated service delivery.

Objective: This study aimed to assess the performance and the results of bidirectional TB/DM screening, diagnosis of co-morbidity, and enrollment in treatment in 7 health operational districts in 5 provinces in Cambodia.

Methods: The retrospective study reviewed patient records of 6,463 DM patients and 8,403 TB patients who received treatment between July 2016 and February 2019 in 7 referral hospitals and 113 health centers.

Results: Forty percent of DM patients were screened for TB, and 55\% of TB patients were screened for DM. Of the screened DM patients, $4.6 \%$ were diagnosed with TB. Of screened TB patients, 3.7\% were diagnosed with DM. All DM patients diagnosed with TB were enrolled in TB treatment and 95\% of TB patients diagnosed with DM began receiving treatment for DM.

Conclusion: This is the first study examining TB/DM co-morbidity and coordinated service delivery in Cambodia. The gaps in the performance of bidirectional screening suggest areas for further intervention. To increase the rate of bidirectional screening, provider compliance with standards needs to be improved by strengthening providers competencies. Strengthened data collection and reporting systems will also contribute to increasing provider accountability. Secondly, the current structure of TB and DM service delivery with TB services only available from the public sector and public DM services only available at the referral level creates a challenging environment for effective referrals and coordinated care and should be reconsidered. In addition to improved coordination between the public and private sectors, expansion of public DM services to health centers and to the community level warrants exploration. Finally, the increased focus needs to be given to addressing the high levels of pre-diabetes. Cambodia has a limited window of opportunity to build capacity and develop systems to effectively manage TB/DM co-morbidity.
\end{abstract}

Keywords: Diabetes mellitus; Tuberculosis; Integration of services; Bi-directional screening; Treatment outcomes

Corresponding author: Soy Ty Kheang, Center for Health and Social Development (HSD), Phnom Penh, Cambodia, Tel: + 85517988387; E-mail: ksoyty@urc-chs.com

Received date: July 10, 2019; Accepted date: July 23, 2019; Published date: August 02, 2019

Citation: Kheang ST, Theang H, Eam KK, Eang MT, Kong S, Loun C, et al. (2019) Bidirectional Screening of Diabetes Mellitus and Tuberculosis in Cambodia. J Trop Dis 7:326.

Copyright: ( 2019 Kheang ST, et al. This is an open-access article distributed under the terms of the Creative Commons Attribution License, which permits unrestricted use, distribution and reproduction in any medium, provided the original author and source are credited. 


\section{INTRODUCTION}

The association between Tuberculosis (TB) and Diabetes Mellitus (DM) is well-established. People with DM have impaired cell-mediated immunity, micronutrient deficiency, pulmonary microangiopathy and renal insufficiency which increase their risk of TB [1]. As a result, they have triple the risk of developing active TB than people without DM [2]. Patients with dual disease may have worse treatment outcomes in terms of smear and culture conversion, TB treatment failure, and TB case fatality compared to those non-diabetic patients [3]. In 2015, 10.6\% (6.8-14.8) of global TB deaths among HIV-negative individuals were due to DM [4]. At the same time, TB may impair glucose tolerance, increasing the risk of developing DM [5].

Cambodia is among the top 30 countries with the highest burden of TB with an incidence rate of 326 per 100,000 population in 2018 [6]. While data on the prevalence of DM is limited, a 2010 survey found diabetes prevalence among people aged 25-64 to be 2.9\% [7]. A repeat survey in 2016 found a prevalence of $9.6 \%$ [8]. Recognizing the need to increase focus on TB and DM co-morbidity, the Cambodian Ministry of Health $(\mathrm{MOH})$ developed the Draft Guideline For Management of Diabetes Mellitus-Tuberculosis Co-morbidity in 2014 [9]. However, no studies have been published on the implementation of integrated TB and DM activities or their results. We conducted a retrospective study in 7 health operational districts in 5 provinces to assess the performance and the results of the bidirectional screening.

\section{METHODOLOGY}

This retrospective study assesses the prevalence of TB among DM patients and DM among TB patients identified through bidirectional screening at public health facilities during July 2014-February 2019. The screening was conducted as part of a pilot project implemented by the Center for Health and Social Development (HSD) in collaboration with the MOH's National Center for TB Control (CENAT) and the Department of Preventive Medicine (DPM). The project operationalized the Draft Guideline For Management of Diabetes MellitusTuberculosis Co-morbidity, including the referral form and patient counseling guidelines, at the facility level. The pilot project involved 7 referral hospitals and 113 health centers in the provinces of Kamcham (Choeung Prey operational district), Prey Veng (Pearaing operational district), Siem Reap (Siem Reap and Sotnikum operational districts), Takeo (Daunkeo and Kirivong operational districts), Tbaung Khmom Province (Memut operational district). The operational districts were selected because the referral hospital in the district had an existing diabetes clinic.

To support the implementation of bidirectional screening, health providers at participating facilities received training on bidirectional screening, diagnosis, and treatment of patients with both TB and DM, referral between TB and DM service providers, infection control, and monitoring and reporting. The project also worked with CENAT and DPM to pilot revised TB and DM patient records that integrated bidirectional screening information. This study reviewed data from the TB and DM patient records.

\section{Screening DM patients for TB}

DM patients receiving services at referral hospital diabetes clinics were screened for TB according to the CENAT guidelines. Patients were asked if they had experienced a cough for more than two weeks at the time of DM diagnosis as well as during subsequent routine clinical visits. TB screening findings were noted in the Diabetes Registration Form. Patients with suspected $\mathrm{TB}$ were referred to $\mathrm{TB}$ wards for diagnosis using sputum smear examination or, when smear-negative, chest radiography, and for TB treatment initiation.

\section{Screening TB patients for DM}

TB patients receiving services from referral hospitals and health centers were screened for hyperglycemia at TB treatment initiation and every two months at follow-up visits. DM screening results were included in the revised TB registration book which was being piloted by the NTP in the participating facilities. Patients with a fasting blood glucose (FBG) $\geq 126$ $\mathrm{mg} / \mathrm{dl}$ ) or random blood glucose $(\mathrm{RBG}) \geq 200 \mathrm{mg} / \mathrm{dl}$ were referred to diabetes clinics for confirmation and care, where they are tested using HbA1c.

\section{Case management of DM/TB patients}

Patients diagnosed with both TB and DM had their glucose levels checked during scheduled TB follow-up visits and received counseling on lifestyle changes and self-management. Treatment regimens were not changed as a result of co-morbidity.

\section{RESULTS}

The results of the bidirectional screening are summarized in Table 1. 6,463 DM patients were enrolled in the study, of whom a majority were female (63.5\% versus $36.5 \%$ male). Of the DM patients receiving services between July 2016 and February 2019, 2,580 (39.9\%) were screened for TB. Of the screened cases, 715 (27.7\%) were suspected of having TB and referred for diagnosis. 118 DM patients were diagnosed with $\mathrm{TB}$, providing a $4.6 \% \mathrm{~TB}$ prevalence among DM patients screened for TB. All DM patients diagnosed with TB were begun on treatment in a health facility and received regular follow-up for TB treatment by community-level providers of Directly Observed Therapy, Short Course (DOTS).

Of the 8,403 TB patients, $55 \%$ were male and $45 \%$ were female. 4,635 (55\%) were screened for DM and 417 with high blood sugar were referred to diabetes clinics for confirmation of DM diagnosis. $172 \mathrm{~TB}$ patients were diagnosed with DM, providing a DM prevalence of $3.7 \%$ among TB patients screened for DM. Almost all (95\%) of TB patients diagnosed with DM enrolled in DM services and received DM treatment. Testing TB patients for DM also identified 834 patients with pre-diabetes. 
Table 1: Results of bidirectional screening by 7 referral hospitals and 113 health centers, July 2016-February 2019.

\begin{tabular}{ll}
\hline Indicator & Number (\%) of patients \\
\hline Number of DM patients & 6,463 \\
\cline { 2 - 2 } & $2359(36.5 \%)$ male \\
\cline { 2 - 2 } & $4104(63.5 \%)$ female \\
\hline $\begin{array}{l}\text { Number of DM patients screened for } \\
\text { TB }\end{array}$ & \\
\hline
\end{tabular}

Number of DM patients screened for 715 (27.7\%)

TB with suspected TB

Number of DM patients screened for 118 (4.6\%)

$\mathrm{TB}$ who were diagnosed with TB

Number of DM patients diagnosed 118 (100\%)

with TB who initiated TB treatment

\begin{tabular}{ll}
\hline Number of TB patients & 8,403 \\
\cline { 2 - 2 } & \begin{tabular}{l}
$4622(55 \%)$ male \\
\hline $781(45 \%)$ female
\end{tabular} \\
& Referral hospital: 2,622 \\
Health center: 5,781 \\
\hline
\end{tabular}

Number of TB patients screened for 4,635 (55\%)

$\mathrm{DM}$

Referral hospital: 1,476

Health center: 3,159

Number of TB patients screened for 834 (17.9\%)

DM who were identified as having pre-

diabetes

Number of TB patients screened for 417 (9.0\%)

DM with suspected DM

Number of TB patients screened for $172(3.7 \%)$

DM diagnosed with DM

Number of TB patients diagnosed with 163 (95\%)

DM who enrolled in DM services

\section{DISCUSSION}

This retrospective review assessed the results of bidirectional screening conducted at 7 referral hospitals and 113 health centers in 5 provinces of Cambodia. Data for 6,463 DM patients and 8,403 TB patients were analyzed by the study. Similar to national data, DM patients were more likely to be women [7] and TB patients were more likely to be men [10]. The study found a DM prevalence of 3.7\% among patients with TB, higher than the 2010 prevalence of $2.9 \%$ in the general population [7] but lower than the 2016 prevalence of $9.6 \%$ [8]. The study also found a higher incidence of TB among DM patients than the general population, 4,600 compared to 326 per 100,000 population [6] respectively. These findings highlight the negative interaction between TB and DM and also suggest that targeting $\mathrm{TB}$ case finding at $\mathrm{DM}$ patients may be an effective approach to TB control in Cambodia.

The gaps in the performance of bidirectional screening in the pilot areas suggest some areas where additional efforts are needed. Despite receiving training and support, the health providers screened only $39.9 \%$ of DM patients for TB and $55 \%$ of TB patients for DM (56\% at referral hospitals and $55 \%$ at health centers). The challenge of motivating the health workforce to take on the additional screening task has also been documented in other countries [11,12]. Effective models that ensure that provider complies with standards and apply the skills learned to service delivery need to be identified and scaled up.

A second gap is the referral process for TB patients diagnosed with DM. TB services, which are free, are provided only by the public sector, involving community-level DOTS providers, health centers, and referral hospitals. As a result, accessing TB diagnosis and treatment is relatively well supported. In contrast, public sector DM services are not included in the basic package of health care provided by health centers but are only available at referral hospitals. As a result, while DOTS workers or health centers can provide screening, health education, and counseling, they must then refer patients to the DM clinic at the referral hospital for confirmation and care. In this study, this may have resulted in the loss of some TB patients to DM follow-up. Potential barriers to TB patients seeking confirmation of DM diagnosis and care include transportation and payment for services at the referral hospital. Without effective referral mechanisms that include feedback to the lower level, monitoring the effectiveness of referrals is difficult.

Expanding the availability of DM services to the health center level would facilitate not only confirmation of DM diagnosis but also ongoing care and support. Such an approach might also be considered in Cambodia. Modeling of the epidemiological and economic consequences of DM in Cambodia found that achieving full coverage of patients requiring Oral Anti-diabetic Drugs (OAD), for example through free or subsidized access to the drugs at health centers, would avert 25,000 deaths from 2008 to 2028 while increasing cost-effectiveness [13]. Offering DM services at health centers would also strengthen the integration of TB and DM services.

TB patients diagnosed with DM may also be lost to follow up in the public sector because they shift to the private sector for care. In a household survey of 709 individuals with DM or hypertension, only $20 \%$ of respondents said public facilities were their usual source of care, compared to $52 \%$ for private clinics or pharmacies, and $22 \%$ who used multiple sources. Although they are more expensive, private providers are considered more trustworthy and are more likely to have medicines available; public facilities only provide medicines for three days at a time [14]. Due to the limited availability of DM services in public facilities and the high cost of private sector services, patients incur significant expenses: the survey found that $11 \%$ of individuals with DM and hypertension were indebted due to health-related expenses, even though half belonged to a social health protection scheme [14]. 
Regardless of the source of care, very low proportions of DM patients in Cambodia reach their treatment targets [14,15], which in turn may increase their risk of TB [16-18]. The reliance on the private sector for diabetes care combined with limiting TB services to the public sector presents a unique challenge for integrating service delivery. As patients obtain services and medicines from several different sources, tracking and sharing patient information and ensuring coordinated care between public TB service providers and private DM service providers becomes critical.

The high prevalence of pre-diabetes in TB patients (19\%) reinforces projections of increasing $\mathrm{DM}$ prevalence rates in Cambodia [16,19]. In addition to policy and environmental interventions, pre-diabetes can be addressed through individuallevel information and counseling on nutrition and physical activity. One strategy for DM education to engage community health workers who are already working in TB control [20]. Cambodia's community-level workers have also been found eager to engage in diabetes prevention but would require comprehensive training [21].

The data for the study was drawn from the existing national health information system, currently undergoing revisions to incorporate fields for TB screening on DM records, and fields for DM screening on TB records. As such, the findings provide a baseline for ongoing monitoring of $\mathrm{DM} / \mathrm{TB}$ co-morbidity in the country. CENAT is also currently piloting an updated TB registry. Going forward, DM and TB records may be further revised to incorporate information on the management of the co-morbidity, such as the results of TB patient glucose monitoring every two months.

The study did not examine what caused the performance gaps in bidirectional screening, with the discussion extrapolating from other similar studies. Analysis of data disaggregated by sex and age would have provided additional insight into who is at risk and who are receiving the services they need. Further research is also needed on the health system factors that influence integrated DM/TB service delivery, such as health workforce skills and motivation, the availability and distribution of medicines and equipment, health financing coverage of integrated services. The study also did not assess the quality of integrated $\mathrm{DM} / \mathrm{TB}$ care. Information on whether patients are being counseled and treated according to the DM/TB guidelines and the effectiveness of the referral process not only between public DM and TB service providers but also between public and private providers would help guide efforts to strengthen service delivery.

Efforts to integrate TB and DM services in Cambodia have only begun in the past five years, after the introduction of the 2014 guidelines for the management of DM/TB co-morbidity, and implementation remains at an early stage. The 2016 Technical Guidelines on Tuberculosis Control incorporated guidance on $\mathrm{DM} / \mathrm{TB}$ co-morbidity. As national standards for TB and for DM are updated to incorporate guidelines on DM/TB co-morbidity, the feasibility and cost-effectiveness of different diagnostic approaches will need to be carefully considered. The gold standard for DM diagnosis is the HbA1c, [11] but current resources are barely sufficient to provide health centers with glucometers. The country may also need to consider when and how to shift from sputum smear examination and chest radiography for $\mathrm{TB}$ diagnosis to the use of nucleic acid amplification, such as Xpert, in order to capture all cases [11]. Decentralization of DM diagnosis and care, health workforce training, and institutionalization of health information system changes are additional strategies to ensure that Cambodia establishes integrated systems for DM and TB co-management before DM becomes an overwhelming challenge.

\section{CONCLUSION}

The retrospective review of the performance and results of bidirectional screening found higher levels of DM and of TB among $\mathrm{TB}$ and $\mathrm{DM}$ patients respectively than in the general population. As Cambodia works to control TB, screening DM patients will be an effective strategy. The efforts to establish integrated DM/TB services are also timely given the currently low prevalence of DM. This provides a window of opportunity to build capacity and develop systems to effectively manage the co-morbidity. The findings from the pilot of bidirectional screening can inform the further development of the integrated $\mathrm{DM} / \mathrm{TB}$ program in Cambodia.

Three bottlenecks identified in the discussion about the pilot project findings warrant further exploration. The current policy on providing only three days worth of medicines at public facilities should be revisited. The combination of limited availability of DM medicines with the challenges of accessing care at DM clinics at referral hospitals is resulting in patients cobbling together care from multiple sources, with the result that their DM goes unmanaged, increasing their risk for TB.

Second, given the reliance of DM patients on private clinics and pharmacies, stronger referral systems need to be developed, particularly for TB services which are only available in the public sector. Private providers should be engaged in joint training efforts on $\mathrm{DM} / \mathrm{TB}$, routinely screen their $\mathrm{DM}$ patients for $\mathrm{TB}$, and use the $\mathrm{DM} / \mathrm{TB}$ referral form to share information with the patient's TB provider.

Finally, given the increasing prevalence of DM, expansion of DM services to the community-level is inevitable. Increased availability of public DM services would also enable improved coordination between DM and TB service delivery, as individuals receive information on both diseases from the community health worker, and access care for both diseases from their preferred source of care. Given the limited availability of resources, careful planning is needed on when and how this should take place. At a minimum, training of DOTS workers should begin to incorporate content on diabetes and DM/TB co-morbidity.

\section{ACKNOWLEDGEMENT}

The Coordinated Diabetes/TB Services in Cambodia Project (Phases I and II) was funded by the World Diabetes Foundation and implemented by the Center for Health and Social Development in collaboration with the National Center for TB Control and the Department of Preventive Medicine at the Ministry of Health. 


\section{REFERENCES}

1. Kapur A, Harries AD, Lönnroth K, Bygbjerg C, Lefèbvre P. Diabetes and Tuberculosis-Old associates posing a renewed public health challenge. Europ Endocrinol. 2009;5:10-12.

2. Jeon CY, Murray MB. Diabetes mellitus increases the risk of active tuberculosis: A systematic review of 13 observational studies. PLoS Med. 2008;5:e152.

3. Baker MA, Harries AD, Jeon CY, Hart JE, Kapur A. The impact of diabetes on tuberculosis treatment outcomes: a systematic review. BMC Med. 2011;9:81.

4. GBD Tuberculosis Collaborators. The global burden of tuberculosis: results from the Global Burden of Disease Study 2015. Lancet Infect Diseases. 2018;18:261-84.

5. Niazi AK, Kalra S. Diabetes and tuberculosis: A review of the role of optimal glycemic control. J Diabetes Metabol Disord. 2002;11:28.

6. World Health Organization. Global Tuberculosis Report, Geneva: World Health Organization. 2018.

7. Oum S, Prak PR, Khuon EM, Mey V, Aim S. Prevalence of noncommunicable disease risk factors in Cambodia: STEPS survey country report. Phnom Penh: University of Health Sciences and the Ministry of Health. 2010.

8. Ministry of Health. National STEPS 2016 survey (draft report), Phnom Penh: Ministry of Health. 2016.

9. Ministry of Health. Draft Guideline for management of Diabetes Mellitus-Tuberculosis Co-morbidity, Phnom Penh: Ministry of Health. 2014.

10. World Health Organization. Global Tuberculosis Report, Geneva: World Health Organization. 2018.

11. Harries AD, Kumar AMV, Satyanarayana S, Lin Y, Zachariah R, Lönnroth K, et al. Diabetes mellitus and Tuberculosis: Programmatic management issues. Int J Tuberculosis Lung Dis. 2015;19:879-886.
12. Lekoubou A, Awah P, Fezeu L, Sobngwi E, Kengne AP. Hypertension, Diabetes Mellitus and task shifting in their management in Sub-Saharan Africa. Int J Environ Res Public Health. 2010;7:353-363.

13. Flessa S, Zembok A. Costing of diabetes mellitus type II in Cambodia. Health Econ Rev. 2014;4:24.

14. Bigdeli M, Jacobs B, Men CR, Nilsen K, Van Damme W, Dujardin B. Access to treatment for Diabetes and Hypertension in rural Cambodia: Performance of existing social health protection schemes. PLoS ONE. 2016;11:e0146147.

15. Raguenaud ME, Isaakidis P, Reid T, Chy S, Keuky L, Arellano G, et al. Treating 4,000 diabetic patients in Cambodia, a highprevalence but resource-limited setting: a 5-year study. BMC Med. 2009;7.

16. Odone A, Houben RMGJ, White RG, Lonnroth K. The effect of diabetes and undernutrition trends on reaching 2035 global tuberculosis targets. Lancet Diabetes Endocrinol. 2014;2:754-764.

17. Leung CC, Lam TH, Chan WM, Yew WW, Ho KS, Leung GM, et al. Diabetic control and risk of tuberculosis: a cohort study. Am J Epidemiol. 2008;167:1486-1494.

18. Kumpatla S, Sekar A, Achanta S, Sharath BN, Kumar AMV, Harries AD, et al. Characteristics of patients with diabetes screened for tuberculosis in a tertiary care hospital in South India. Public Health Action. 2013;3:S23-S28.

19. International Diabetes Federation. Cambodia Country Report 2017 and 2045. 2017.

20. Sullivan T, Ben Amor Y. The Co-Management of Tuberculosis and Diabetes: Challenges and opportunities in the developing world. PLoS Med. 2012;9:e1001269.

21. Wagner J, Keuky L, Fraser-King L, Kuoch T, Scully M. Diabetes prevention through village health support guides in Cambodia: A qualitative investigation of opportunities and challenges. J Commu Med and Health Edu. 2015;5:347. 